\title{
Efeitos Genéticos e Fenotípicos sobre Características de Produção e Reprodução de Ovinos Deslanados no Distrito Federal
}

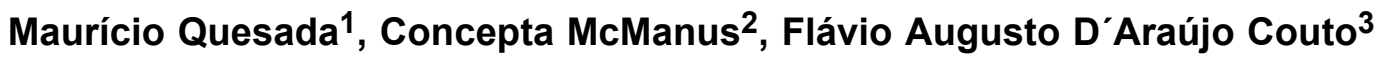

\begin{abstract}
RESUMO - O objetivo deste trabalho foi estimar efeitos genéticos e fenotípicos na criação de ovinos deslanados e mestiços em um criatório no Distrito Federal. Foram analisados os efeitos de sexo, tipo de parto, mês e ano de nascimento sobre peso ao nascer, aos 30 , 120 e 210 dias de idade, intervalo de partos (IDP) e idade ao primeiro parto (IPP) de 2337 nascimentos / parições em um rebanho comercial para produção de carne, das raças Morada Nova (MN), Santa Inês (SI) e mestiços Texel x Morada Nova (T x MN), controlado durante 14 anos (1986 a 1999). Os dados da raça MN foram analisados usando um Modelo Animal Univariado no programa MTDFREML para determinar herdabilidade e repetibilidade bem como correlações genéticas e fenotípicas entre as características. O número médio de crias por ovelha foi de 1,25 cordeiros. Os nascimentos ocorreram durante todo o ano, porém com maior índice de cordeiros nascidos (77\%), entre os meses de março a setembro, indicando possível influência da extensão do dia ou disponibilidade de alimentação sobre a eficiência reprodutiva. As herdabilidades diretas para peso ao nascer, pesos com 30, 120 e 210 dias foram de 0,15; 0,11; 0,07; e 0,21, respectivamente, para a raça Morada Nova. As correlações genéticas entre os pesos variaram de 0,19 a 0,64 . A herdabilidade para intervalo de partos foi de 0,17 e de 0,93 para número de crias. Os efeitos da raça e do peso ao nascer foram altamente significativos, para todos os pesos estudados. O tipo de parto influenciou o peso ao nascer e aos 30 e 210 dias. Os efeitos do número de partos e do mês de nascimento mostraram influências significativas sobre as características avaliadas.
\end{abstract}

Palavras-chave: correlações, herdabilidade, Morada Nova, Santa Inês, repetibilidade, pesos

\section{Estimation of Genetic and Phenotypic Parameters for Brazilian Hair Sheep in Central Brazil}

\begin{abstract}
This study aims to estimate genetic and phenotypic parameters for the Morada Nova, Santa Inês e crossbred Texel x Morada Nova in Federal District of Brazil. Data were available on 2337 lambings over a period of 14 years (1986 to 1999) from a commercial holding for meat production. The herd had no fixed breeding season. The effects of sex, number of lambs per lambing, month and year of lambing were determined for weights at birth, 30,120 and 210 days of age as well as lambing interval and age at first lambing using analysis of variance procedures in the SAS program. Heritability, repeatability as well as genetic and phenotypic correlations for the Morada Nova sheep in the herd were estimated using MTDFREML. The generation interval was 952 days, with a mean litter size of 1.25 lambs. Lambings were distributed throughout the year, but 77\% occurred between March and September, indicating a possible influence of day length or food availability on reproductive traits. Direct heritabilities for birth weight, weight at 30 , 120 and 210 days were, respectively, $0.15,0.11,0.07$, and 0.21 for Morada Nova sheep. The correlations between these traits were positive (0.19 at 0.64$)$. The heritability for lambing interval was 0.17 and 0.93 for number of lambs per litter. The results indicate that the breed and birth weight were highly significant for all weights studied. Lambing number and birth month affect all the traits examined.
\end{abstract}

Key Words: correlation, heritability, Morada Nova, repeatability, Santa Ines, weights

\section{Introdução}

No Nordeste, encontra-se o segundo maior rebanho ovino do país, cerca de 6.597 .796 cabeças (IBGE,1995). Os criadores são proprietários de plantéis pequenos e de várias raças. No Ceará, por exemplo, cerca de 66 a $70 \%$ do rebanho é constituído por animais Sem Raça Definida (SRD) e o número de animais puros é, geralmente, bastante reduzido, mas na raça Santa Inês chega a $18 \%(\mathrm{CNPq}, 1998)$.
A Região Centro-Oeste ocupa o terceiro lugar em população ovina, contando com cerca de 439.683 cabeças (IBGE, 1995). No Distrito Federal, há poucos criadores, porém, com elevado número de animais em cada rebanho (de 100 a 150 cabeças e alguns com mais de 500), normalmente com preponderância da raça Santa Inês (CNPq, 1999).

O rebanho do Distrito Federal está direcionado para a produção de carne como exploração comercial. São criados animais com padrão racial desejável para

\footnotetext{
1 Aluno mestrado, Faculdade de Agronomia e Medicina Veterinária, Universidade de Brasília. E.mail: quesada@unb.br

2 Professora, Faculdade de Agronomia e Medicina Veterinária, Universidade de Brasília. E.mail: concepta@unb.br
}

3 Eng.-Agr., Sítio Campo Alegre, PAD - DF, Brasília, DF. E.mail: fcouto@cnpq.br 
este tipo de exploração, ocorrendo, ainda, como fonte de renda alternativa, venda de reprodutores, tanto para o mercado local, quanto para as áreas próximas.

Destaca-se que a escolha de raças adequadas às condições ambientais locais é um dos fatores preponderantes para o sucesso de um sistema de criação economicamente viável (Barbosa et al., 1995).

A alta diversidade de raças existentes no país passíveis de serem adaptadas às condições do Planalto Central demonstra a necessidade permanente de estudos direcionados para o setor. Os resultados, entretanto, devem ser comparados com os dados obtidos com os animais criados nas diversas áreas de criação, indicando as possibilidades de êxito de um programa de melhoramento genético (McManus \& Miranda, 1998).

No Distrito Federal, além de estudos direcionados para obtenção de parâmetros genéticos e fenotípicos, dos efeitos dos fatores ambientais, visando o conhecimento do comportamento das raças a serem envolvidas, assim como, os diferentes níveis de adaptação desses animais introduzidos no rebanho local, deve-se, ainda, estudar os ovinos para avaliação do seu potencial como opção para diversificar a oferta de proteína animal, para o mercado local (McManus \& Miranda, 1996).

O desenvolvimento populacional do Distrito Federal e regiões adjacentes, sobretudo pela característica de parte da população de Brasília, concentrando grandes contingentes oriundos do Nordeste, do Sul, e do exterior devido principalmente a seus hábitos alimentares, determinam considerável demanda para este tipo de produto animal (CNPq, 1999).

Este estudo objetivou estimar os efeitos sexo, raça, tipo de parto, número de crias, mês e ano de nascimento e peso da ovelha sobre os pesos ao nascer, 30, 120 e 210 dias de idade, intervalo de parto, idade ao primeiro parto e número de crias nascidas das raças Morada Nova (MN), Santa Inês (SI) e mestiços de Texel x MN (T x MN) criados no DF, bem como os parâmetros genéticos e fenotípicos da raça Morada Nova (herdabilidade, repetibilidade e correlações genéticas e fenotípicas) para estas características.

\section{Material e Métodos}

O local do estudo fica a $15^{\circ} 47^{\prime} \mathrm{S}$ e $47^{\circ} 56^{\prime} \mathrm{W}$ Gr. na rodovia DF-100, Colônia Agrícola Itapeti, Distrito Federal. O clima da região é do tipo AW pela classificação de Köppen, com temperatura média anual de $21,1^{\circ} \mathrm{C}$, tendo $-1,6$ e $34^{\circ} \mathrm{C}$ como mínima e máxima absoluta, respectivamente. A precipitação média anual é de $1.578,5 \mathrm{~mm}$ e a média anual de umidade relativa do ar, de $68 \%$. O clima caracteriza-se por duas estações bem definidas, uma das águas, em que ocorre quase toda a precipitação (de outubro a abril), e outra seca, quase sem chuvas (de maio a setembro).

Os animais foram mantidos em regime extensivo de pasto (aproximadamente 20 ha de pastagem) com capim (Andropogon gayanus) e cana desintegrada, complementada com milho no período da seca, com fornecimento de sais minerais efetuado à vontade.

Foi realizado controle regular de endoparasitas com a aplicação de vermífugos. Os animais foram vacinados contra febre aftosa, carbúnculo sintomático, gangrena gasosa e enterotoxemia. Os animais do rebanho são pesados, identificados e anotadas todas as ocorrências com relação ao rebanho, bem como casos de perdas, mortes e outras ocorrências. A monta ocorreu durante todo o ano, e a desmama feita quando os animais apresentavam cerca de três meses de idade.

As cordeiras procedentes de matrizes com boas características raciais foram selecionadas para reprodução, e os cordeiros que não apresentaram condições para reprodução foram vendidos para abate com aproximadamente 6 meses de idade. As pesagens foram feitas ao nascer, aos 7, 15, 30 e 120 dias (desmama) e, novamente, aos 210 dias, próximo ao período de acasalamento das fêmeas de primeira cria.

Realizado o expurgo dos dados do rebanho geral, devido à falta de informações necessárias ao modelo adotado para avaliação (na série histórica de 14 anos), obtiveram-se 2337 animais com informações passíveis de serem utilizadas.

Os dados foram divididos em dois grupos para análise:

1) Referentes ao rebanho total (período de 1986 a 1999), com 2337 animais SI (785), MN (1312) e Texel x MN (240). Foi realizada análise dos dados usando os procedimentos Correlações (CORR) e Análise de Variância (GLM) do SAS (1995). Utilizou-se o mesmo modelo matemático para todas as características, variando-se apenas os efeitos fixos e covariáveis considerados. Os efeitos de grupo genético (SI, TxMN, MN), ordem do parto $(1, \ldots, 8)$, mês (jan, .., dez) e ano de nascimento $(1986, \ldots, 1999)$ foram investigados para todas as características (pesos ao nascer, aos 30, 120 e 210 dias de idade, número de crias por parto, idade ao primeiro parto (IPP) e o intervalo de parto (IEP)). O peso ao nascer foi incluído no modelo como covariável para pesos aos 30, 120 e 210 dias de idade. O número de cordeiros por parto foi incluído como fator fixo em todas as 
características, exceto quando este fator foi analisado como característica.

2) Usando os dados das características do rebanho Morada Nova, foram realizados os cálculos de herdabilidade, repetibilidade, correlações genéticas e fenotípicas adotando-se o programa MTDFREML (Multiple Trait Derivate Free Restricted Maximum Likelihood), segundo Boldman et al. (1995) ajustando-se a equação:

$$
\mathrm{Y}=\mathrm{Xb}+\mathrm{Z}_{1} \mathrm{a}+\mathrm{Z}_{2} \mathrm{~m}+\mathrm{Z}_{3} \mathrm{pe}+\mathrm{e}
$$

em que: $Y$ é um vetor (Nx1) de observação do animal; $b$, o vetor de efeitos fixos (sexo, mês e ano de nascimento e tipo de parto para os pesos e mês e ano de parição, ordem da pariçaõ e tipo de parto para as características de reprodução) no modelo, associado com o matriz de incidência $X$; a, o vetor dos efeitos genéticos diretos, associado com a matriz de incidência $\mathrm{Z}_{1} ; \mathrm{m}$, o vetor dos efeitos genéticos maternos, associado com a matriz de incidência $Z_{2}$; pe, o vetor dos efeitos de ambiente materno permanentes, associado com a matriz de incidência $\mathrm{Z}_{3}$; e $e$, o vetor de resíduos aleatórios.

\section{Resultados e Discussão}

Os resultados das análises de variância para intervalo de partos (IEP), idade ao primeiro parto (IPP), número de partos (NP), peso ao nascer (PN), e pesos aos 30,120 e 210 dias de idade de ovinos das raças Morada Nova e Santa Inês, assim como dos mestiços Texel x Morada Nova estão apresentados na Tabela 1.

Observa-se que o ano de nascimento afetou todas as características, devido, provavelmente, às variações de ambiente e manejo do rebanho durante os anos. Peso ao nascer, como covariável, afetou os pesos aos 30,120 e 210 dias, sendo que animais mais pesados ao nascer demonstraram a tendência de manter esta superioridade até 210 dias. O sexo afetou peso ao nascer e aos 120 dias. O tipo de parto não foi significativo para IEP, tendo sido significativo $(\mathrm{P}<0,05)$ para idade do primeiro parto (IPP) e para todos os pesos estudados $(\mathrm{P}<0,01)$, exceto $\mathrm{P} 120$. A raça influenciou significativamente os pesos dos cordeiros aos todos as idades estudadas bem como idade ao primeiro parto $(\mathrm{P}<0,01)$.

No Brasil, a influência dos seguintes fatores foi verificada: tipo de parto Fernandes et al. (1993); ano de nascimento e tipo de parto, Figueiredo et al. (1985); sexo e ano de nascimento, Costa et al. (1990); sexo, ano de nascimento e tipo de parto, Silva et al. (1998); sexo e ano de nascimento, Roda et al. (1995). Não houve efeito da idade da mãe sobre pesos, contrastando com os resultados obtidos por Fernandes et al. (1985) e Roda et al. (1995).

$\mathrm{Na}$ Tabela 2 são apresentadas médias do peso ao nascer, aos 30, 120 e 210 dias de idade, do intervalo

Tabela 1 - Resumo das análises de variância para intervalo de partos (IEP), idade ao primeiro parto(IPP), número de cordeiros por parto (NC), peso ao nascer (PN), peso aos 30 (P30), 120 (P120) e 210 (P210) dias de idade em ovinos das raças Morada Nova e Santa Inês, e mestiços Texel x Morada Nova.

Table 1 - Summary of analysis of variance for birth weight (BW), weights at 30 (W30), 120 (W120) and 210 (W210) days of age, lambing interval (LI), number of lambs per parturition (NL) and age at first lambing (AFL) for Morada Nova, Santa Ines and Texel $x$ Morada Nova sheep raised in the Federal District, Brazil

\begin{tabular}{|c|c|c|c|c|c|c|c|}
\hline & $\begin{array}{l}\text { IEP } \\
L I\end{array}$ & $\begin{array}{l}\text { IPP } \\
A F L\end{array}$ & $\begin{array}{l}\mathrm{NC} \\
N L\end{array}$ & $\begin{array}{l}\mathrm{PN} \\
B W\end{array}$ & $\begin{array}{l}\mathrm{P} 30 \\
W 30 \\
\end{array}$ & $\begin{array}{l}\mathrm{P} 120 \\
W 120 \\
\end{array}$ & $\begin{array}{l}\mathrm{P} 210 \\
W 210\end{array}$ \\
\hline Sexo & & & & $* *$ & $\mathrm{~ns}$ & $* *$ & $\mathrm{~ns}$ \\
\hline Sex & & & & & & & \\
\hline $\begin{array}{l}\text { Ano } \\
\text { Year }\end{array}$ & $* *$ & $* *$ & $* *$ & $* *$ & $* *$ & $* *$ & $* *$ \\
\hline $\begin{array}{l}\text { Mês } \\
\text { Month }\end{array}$ & $* *$ & $\mathrm{~ns}$ & $\mathrm{~ns}$ & $* *$ & $* *$ & $\mathrm{~ns}$ & $\mathrm{~ns}$ \\
\hline $\begin{array}{l}\text { Ordem do parto } \\
\text { Lambing order }\end{array}$ & $* *$ & $\mathrm{~ns}$ & $* *$ & $* *$ & $\mathrm{~ns}$ & $\mathrm{~ns}$ & $\mathrm{~ns}$ \\
\hline $\begin{array}{l}\text { Tipo do parto } \\
\text { Number of lambs }\end{array}$ & $\mathrm{ns}$ & $*$ & & $* *$ & $* *$ & $\mathrm{~ns}$ & $* *$ \\
\hline $\begin{array}{l}\mathrm{PN} \\
B W\end{array}$ & & & & & $* *$ & $* *$ & $* *$ \\
\hline $\begin{array}{l}\text { Raça } \\
\text { Breed }\end{array}$ & $\mathrm{ns}$ & $* *$ & $\mathrm{~ns}$ & $* *$ & $* *$ & $* *$ & $* *$ \\
\hline
\end{tabular}

\footnotetext{
R. Bras. Zootec., v.31, n.1, p.342-349, 2002 (suplemento)
} 
de partos (IEP), do número de cordeiro por parto (NC) e da idade ao primeiro parto(IPP) de ovinos das raças Morada Nova (MN), Santa Inês (SI) e mestiços Texel x Morada Nova (T x MN). Verifica-se que a raça SI e os mestiços apresentaram pesos bem similares, entretanto, comparando com a raça $\mathrm{MN}$, as diferenças são consideráveis, com relevante vantagem para os mestiços a partir de 120 dias. Essa vantagemé, provavelmente, devida ao vigor híbrido e a vantagem aos 30 dias da raça SI deve-se, possivelmente, ao fato de que esta raça produza mais leite. No entanto, quando os cordeiros começam a pastar, verifica-se que os híbridos passam a ter vantagem. Em relação ao IEP, verifica-se que os mestiços apresentaram maior intervalo de partos comparados com as demais raças, sendo que a raça $\mathrm{MN}$ apresentou o menor IEP.

As médias + erro-padrão do intervalo de partos obtidas neste estudo foram de $284,81+5,17$ $(\mathrm{cv}=65,74 \%), 325,02+7,66(\mathrm{cv}=66,02 \%)$ e 346,58 $+13,83(\mathrm{cv}=66,02 \%)$ dias, para as raças MN, SI e mestiços $\mathrm{T} \times \mathrm{MN}$, respectivamente. Estes intervalos de partos foram considerados muito altos, o que pode ser atribuído à alta variabilidade dos animais utilizados para reprodução e à falta de manejo reprodutivo mais rigoroso para evitar IEP muito altos. Trabalhos desenvolvidos com animais da raça Bergamácia apre- sentaram IEP de 248,94 + 10 dias (McManus \& Miranda, 1996), enquanto Costa et al. (1990) apresentaram 251,47 + 98,11 dias com ovelhas Morada Nova no Estado de São Paulo e o valor de $247+53$ dias foi citado por Combellas (1980) para ovinos tropicais, em geral. Apresentam-se na Tabela 3 as médias estimadas pelos mínimos quadrados por sexo e tipo de parto das características de peso até 210 dias de ovinos das raças Morada Nova, Santa Inês e mestiços Texel x Morada Nova.

Sexo, na maioria das pesquisas com ovinos, demonstra um comportamento distinto, agindo como uma característica que apresenta grande influência no crescimento dos cordeiros, principalmente pelo fato dos machos serem mais pesados do que as fêmeas, apresentando dimorfismo sexual. Estudos com cordeiros Santa Inês e cruzados Texel x Santa Inês e Texel x Bergamácia demonstram que os machos são mais pesados ao nascer do que as fêmeas $(3,80 \times 3,22 \mathrm{~kg}$, respectivamente) e, do mesmo modo, os pesos ao desmame foram maiores para os machos $(31,13 \mathrm{~kg})$ do que para as fêmeas $(26,08 \mathrm{~kg})$ (Furusho et al., 1995).

Os efeitos do tipo de parto sobre o peso ao nascer e crescimento dos ovinos até a desmama nos diversos estudos, tanto no Distrito Federal como no Nordeste, apresentam significativa influência no desenvolvi-

Tabela 2 - Médias dos pesos ao nascer (PN), aos 30 (P30), 120 (P120) e 210 (P210) dias de idade, intervalo de partos (IEP), número de cordeiros por parto (NC) e idade ao primeiro parto (IPP) para as raças Morada Nova, Santa Inês e mestiços Texel x Morada Nova criados no Distrito Federal

Table 2 - Means of birth weight (BW), weights at 30 (W30), 120 (W120) and 210 (W210) days of age, lambing interval (LI), number of lambs per parturition (NL) and age at first lambing (AFL) for Morada Nova, Santa Ines and Texel $x$ Morada Nova sheep raised in the Federal District, Brazil

\begin{tabular}{|c|c|c|c|c|c|c|}
\hline \multirow[t]{2}{*}{$\begin{array}{l}\text { Raças } \\
\text { Breeds }\end{array}$} & \multicolumn{2}{|c|}{$\begin{array}{l}\mathrm{MN} \text { (1312 nascimentos) } \\
\quad M N(1312 \text { births })\end{array}$} & \multicolumn{2}{|c|}{$\begin{array}{l}\text { SI ( } 785 \text { nascimentos }) \\
\text { SI ( } 785 \text { births })\end{array}$} & \multicolumn{2}{|c|}{$\begin{array}{c}\text { TxMN (240 nascimentos) } \\
\operatorname{TxMN}(240 \text { births })\end{array}$} \\
\hline & $\begin{array}{l}\text { Médias } \\
\text { Means }\end{array}$ & $\begin{array}{l}\text { Erro-padrão } \\
\text { Standard error }\end{array}$ & $\begin{array}{l}\text { Médias } \\
\text { Means }\end{array}$ & $\begin{array}{l}\text { Erro-padrão } \\
\text { Standard error }\end{array}$ & $\begin{array}{l}\text { Médias } \\
\text { Means }\end{array}$ & $\begin{array}{l}\text { Erro-padrão } \\
\text { Standard error }\end{array}$ \\
\hline $\begin{array}{l}\mathrm{PN} \\
B W\end{array}$ & 2,36 & 0,02 & 3,07 & 0,02 & 2,84 & 0,04 \\
\hline $\begin{array}{l}\mathrm{P} 30 \\
W 30\end{array}$ & 5,54 & 0,11 & 8,01 & 0,14 & 7,53 & 0,27 \\
\hline $\begin{array}{l}\mathrm{P} 120 \\
W 120\end{array}$ & 16,35 & 0,41 & 20,10 & 0,35 & 21,32 & 0,61 \\
\hline $\begin{array}{l}\mathrm{P} 210 \\
W 210\end{array}$ & 26,26 & 0,62 & 29,84 & 0,53 & 32,99 & 1,09 \\
\hline $\begin{array}{l}\text { IEP } \\
L I\end{array}$ & 284,81 & 5,17 & 325,02 & 7,66 & 346,58 & 13,83 \\
\hline $\begin{array}{l}\mathrm{NC} \\
N L\end{array}$ & 1,37 & 0,01 & 1,27 & 0,01 & 1,25 & 0,02 \\
\hline $\begin{array}{l}\text { IPP } \\
A F L\end{array}$ & 502,27 & 6,92 & 551,13 & 7,24 & 597,17 & 7,93 \\
\hline
\end{tabular}


Tabela 3 - Médias estimadas pelos mínimos quadrados para sexo e tipo de parto para características de peso em ovinos Morada Nova, Santa Inês e mestiços Texel x Morada Nova no Distrito Federal

Table 3 - Least squares means for sex and lambing type, for weight traits in Morada Nova, Santa Ines and crossbred Texel x Morada Nova sheep in the Federal District, Brazil

\begin{tabular}{|c|c|c|c|c|c|c|c|c|}
\hline \multirow[t]{3}{*}{ Efeitos } & \multicolumn{2}{|c|}{$\mathrm{PN}(B W)$} & \multicolumn{2}{|c|}{$\mathrm{P} 30(W 30)$} & \multicolumn{2}{|c|}{$\mathrm{P} 120$ (W120) } & \multicolumn{2}{|c|}{$\mathrm{P} 210(W 210)$} \\
\hline & Média & ep & Média & ep & Média & ep & Média & ep \\
\hline & Mean & se & Mean & se & Mean & se & Mean & se \\
\hline & \multicolumn{8}{|c|}{$\begin{array}{l}\text { Raça - Morada Nova } \\
\text { Breed }\end{array}$} \\
\hline \multicolumn{9}{|l|}{ Sexo } \\
\hline \multicolumn{9}{|l|}{ Sex } \\
\hline $\mathrm{M}$ & 2,37 & 0,04 & 5,58 & 0,01 & 17,15 & 0,02 & 26,20 & 0,03 \\
\hline $\mathrm{F}$ & 2,36 & 0,04 & 5,50 & 0,01 & 16,01 & 0,02 & 25,94 & 0,03 \\
\hline \multicolumn{9}{|c|}{ Tipo de parto } \\
\hline \multicolumn{9}{|c|}{ Lambing type } \\
\hline $\begin{array}{l}\text { Simples } \\
\text { Simple }\end{array}$ & 2,46 & 0,04 & 5,93 & 0,01 & 17,10 & 0,02 & 27,32 & 0,03 \\
\hline $\begin{array}{l}\text { Simple } \\
\text { Duplo }\end{array}$ & 2,17 & 0,01 & 5,52 & 0,02 & 16,05 & 0,03 & 23,17 & 0,04 \\
\hline Double & & & & & & & & \\
\hline Triplo & 1,87 & 0,02 & 4,92 & 0,05 & 13,10 & 0,12 & 22,14 & 0,14 \\
\hline Triple & & & & & & & & \\
\hline \multicolumn{9}{|c|}{$\begin{array}{l}\text { Raça - Santa Inês } \\
\text { Breed }\end{array}$} \\
\hline \multicolumn{9}{|c|}{ Sexo } \\
\hline \multicolumn{9}{|l|}{ Sex } \\
\hline M & 3,12 & 0,03 & 8,10 & 0,01 & 20,60 & 0,02 & 30,45 & 0,02 \\
\hline $\mathrm{F}$ & 3,00 & 0,04 & 7,97 & 0,01 & 19,61 & 0,02 & 29,17 & 0,02 \\
\hline \multicolumn{9}{|c|}{ Tipo de parto } \\
\hline \multicolumn{9}{|c|}{ Lambing type } \\
\hline Simples & 3,12 & 0,03 & 8,35 & 0,01 & 20,59 & 0,03 & 29,88 & 0,02 \\
\hline Simple & & & & & & & & \\
\hline Duplo & 2,90 & 0,01 & 7,11 & 0,02 & 19,11 & 0,01 & 29,49 & 0,03 \\
\hline Double & & & & & & & & \\
\hline Triplo & 1,85 & 0,03 & & & & & & \\
\hline Triple & & & & & & & & \\
\hline \multirow{2}{*}{\multicolumn{9}{|c|}{$\begin{array}{l}\text { Raça - Texel x Morada Nova } \\
\text { Breed }\end{array}$}} \\
\hline & & & & & & & & \\
\hline \multirow{2}{*}{\multicolumn{9}{|c|}{$\begin{array}{l}\text { Sexo } \\
\text { Sex }\end{array}$}} \\
\hline & & & & & & & & \\
\hline $\mathrm{M}$ & 2,88 & 0,01 & 7,63 & 0,02 & 21,92 & 0,03 & 33,92 & 0,04 \\
\hline $\mathrm{F}$ & 2,80 & 0,01 & 7,12 & 0,02 & 21,03 & 0,03 & 32,63 & 0,04 \\
\hline \multicolumn{9}{|c|}{ Tipo de parto } \\
\hline \multicolumn{9}{|c|}{ Lambing type } \\
\hline Simples & 3,00 & 0,01 & 7,82 & 0,02 & 21,96 & 0,02 & 33,86 & 0,03 \\
\hline Simple & & & & & & & & \\
\hline Duplo & 2,50 & 0,05 & 5,89 & 0,04 & 20,06 & 0,04 & 31,66 & 0,04 \\
\hline Double & & & & & & & & \\
\hline Triplo & 2,28 & 0,01 & 5,05 & 0,06 & & & & \\
\hline Triple & & & & & & & & \\
\hline
\end{tabular}

PN - peso ao nascer, peso aos 30 (P30), 120 (P120) e 210 (P210) dias; EP = erro-padrão.

$B W$ - birth weight; weights at 30 (W30), 120 (W120) e 210 (W210) days; SE = Standard error. 
mento dos animais e, a grande maioria, demonstra que animais nascidos de partos simples são mais pesados que os de parto duplo e triplo.

Comparando animais das raças Bergamácia e Santa Inês, McManus \& Miranda (1997), na região de Brasília, observaram que os cordeiros obtidos de parto duplo e triplo foram menores em peso ao nascer e à desmama, sendo para parto simples $2,95 \mathrm{~kg}$; parto duplo $2,12 \mathrm{~kg}$ e parto triplo $1,52 \mathrm{~kg}$. Os pesos foram superiores aos obtidos neste estudo para parto simples $(2,82 \mathrm{~kg})$ e os demais (parto duplo $2,59 \mathrm{~kg}$ e parto triplo $2,15 \mathrm{~kg}$ ) superiores aos dados publicados.

A influência do mês de nascimento sobre os pesos variou com a idade observada. O mês de nascimento, atuando sobre crescimento dos cordeiros, é outro fator importante a ser considerado no desenvolvimento dos animais e, consequentemente, no manejo reprodutivo. No Distrito Federal, por existirem duas estações bem definidas, uma seca e outra chuvosa, verifica-se que a produção de alimentos para os animais sofre grandes variações, diminuindo, além da qualidade, as quantidades de matéria seca disponíveis para os mesmos, influenciando no desempenho das matrizes e no aleitamento dos cordeiros, alterando, assim, seu estado físico.

Os pesos ao nascer tiveram valores menores na época da seca e máximos de outubro a dezembro, quando o final da gestação ocorreu com suplementação alimentar e o aleitamento junto com bons pastos. Nas outras idades os pesos variaram muito entre os meses.

$\mathrm{O}$ ano afetou o número de crias por parto e o intervalo de partos. Com o aumento da idade o intervalo de partos diminuiu, possivelmente devido à seleção de fêmeas. Observa-se a tendência do peso ao nascer dos ovinos deslanados aumentar ao longo dos anos e, igualmente, acréscimo neste e outros pesos ao longo dos anos, talvez devido a melhorias em manejo e sanidade, bem como por seleção e substituição de animais da raça Morada Nova pelos da raça Santa Inês, a partir do ano de 1995.

A idade ao primeiro parto do rebanho foi, em média, 550,19+7,24 dias, sendo influenciada pelo ano de parição, pelo número de cordeiros no primeiro parto e pelo grupo genético da ovelha. Existem diferenças significativas entre os grupos genéticos estudados para esta característica, sendo as ovelhas $\mathrm{MN}$ mais precoces $(502,27+6,92$ dias $)$ e as $\mathrm{T} \times \mathrm{MN}$ mais tardias $(597+7,93$ dias $)$ com as da raça SI intermediárias $(551,13+7,24$ dias $)$. Este resultado está de acordo com Torres et al. (1987), trabalhando com ovelhas africanas nativas de Rwanda.

Observaram-se nascimentos em todos os meses do ano, demonstrando que a raça tem comportamento poliestral nas condições de Brasília. Houve uma diminuição no número de crias por parto entre $1993 \mathrm{e}$ 1997, e um aumento em 1998 e 1999, talvez devido ao melhor manejo e seleção de fêmeas. O número de crias também diminuiu após o quinto parto. Nesta idade, geralmente ocorrem problemas com a alimentação das fêmeas, bem como o aparecimento de problemas reprodutivos. Sendo a monta do rebanho livre, verificou-se que $77 \%$ dos nascimentos ocorreram de março a setembro.

As estimativas de herdabilidade, repetibilidade, correlações genéticas e fenotípicas entre características de peso ao nascer e até aos 210 dias, para os animais Morada Nova, são apresentadas na Tabela 4.

Os valores estimados neste estudo podem ser usados em programas de melhoramento na região de Brasília, onde as condições climáticas são favoráveis. Esses coeficientes foram menores que os obtidos por Silva et al. (1995) os quais, trabalhando com animais da raça Santa Inês, encontraram herdabilidade entre 0,25 e 0,29 .

Tabela 4 - Correlações genéticas ( $r_{G}$ - abaixo da diagonal) e fenotípicas ( $r_{p}$ - acima da diagonal) e herdabilidade direta $\left(\mathrm{h}^{2}{ }_{\mathrm{a}}\right)$, herdabilidade materna $\left(\mathrm{h}^{2} \mathrm{~m}\right)$ e efeito do ambiente permanente $\left(\mathrm{c}^{2}\right)$ para características de peso em animais da raça Morada Nova em um criatório no Distrito Federal.

Table 4 - Genetic (below diagonal) and phenotypic (above diagonal) correlations and direct $\left(h^{2}{ }_{d}\right)$ and maternal $\left(h^{2}{ }_{m}\right)$ heritabilities and permanent environment effects for weight traits in Morada Nova sheep in the Federal District, Brazil

\begin{tabular}{lcccc}
\hline & PN & P30 & P120 & P210 \\
& $B W$ & $W 30$ & $W 120$ & $W 210$ \\
\hline PN & & 0,37 & 0,30 & 0,21 \\
$B W$ & & & \\
P30 & 0,64 & & 0,39 & 0,29 \\
$W 30$ & & & & \\
P120 & 0,43 & 0,30 & & 0,34 \\
W120 & & & & \\
P210 & 0,19 & 0,26 & 0,44 & \\
$W 210$ & & & & \\
$\mathrm{~h}^{2}$ & 0,15 & 0,11 & 0,07 & 0,21 \\
$\mathrm{~h}^{2}{ }^{\mathrm{a}}$ & 0,22 & 0,14 & 0,10 & 0,09 \\
$\mathrm{c}^{2}$ & 0,11 & 0,15 & 0,07 & 0,08 \\
\hline
\end{tabular}

PN - peso ao nascer, peso aos 30 (P30), 120 (P120)e 210 (P210) dias; $\mathrm{c}^{2}=\sigma_{\mathrm{pe}}^{2} / \sigma_{\mathrm{p}}^{2}$

BW-birth weight; weights at 30 (W30), 120 (W120) and 210 (W210) days; $c^{2}=\sigma_{p e}^{2} / \sigma_{p}^{2}$. 
A criação de animais Santa Inês no Ceará apresenta valores de herdabilidade para pesos do nascer até 112 dias variando entre 0,19 e 0,29 e acompanhadas de altas correlações genéticas e fenotípicas entre os diversos pesos (Silva et al., 1995).

Valores de herdabilidades para peso ao nascer $(0,27)$ e para peso aos 112 dias $(0,29)$, obtidos por Silva et al. (1993a), foram mais baixos do que os da Raça Santa Inês (Silva et al., 1993b), que foram 0,48 para peso ao nascer e 0,53 para peso aos 112 dias de idade.

Com animais da raça Bergamácia, McManus \& Miranda (1998) obtiveram valores de médios a altos para herdabilidade $(0,22-0,34)$ dos ganhos entre pesos sucessivos, e baixos para herdabilidades das características reprodutivas $(0,04$ a 0,27$)$. As correlações genéticas entre as características de peso foram, em geral, altas $(>0,65)$, tendo sido para correlação genética de peso ao nascer e na desmama de $0,6+0,15$ e a correlação fenotípica entre estas idades de 0,29+0,05.

Trabalhando com ovinos da raça Morada Nova no Estado do Ceará, Fernandes et al. (1993) estimaram valores de herdabilidade para pesos, do nascer até um ano de idade, variando entre 0,06 e 0,14, inferiores às encontradas.

$\mathrm{Na}$ maior parte dos trabalhos citados acima, os modelos usados foram mais simples, em geral não levando em consideração os efeitos maternais ou de ambiente permanente. Sousa et al. (1999) estudaram o efeito do modelo usado nas estimativas de herdabilidade e concluíram que o modelo completo incluindo estes efeitos é o mais apropriado na estimativa de herdibilidades de características de crescimento, especialmente na época pré-desmama, quando efeitos maternos são importantes. As estimativas encontradas por Sousa et al. (1999) são da mesma magnitude que as deste trabalho, para idades similares.

Os efeitos maternos foram de menor importância após a desmama, como esperado e estão de acordo com outros autores (Sousa et al., 1999; Tosh \& Kemp, 1994).

As estimativas das correlações genéticas das características estudadas foram superiores àquelas encontradas por Silva et al. (1993a) e Sousa et al. (1999), porém inferiores às obtidas por McManus \& Miranda (1998).

As correlações genéticas entre os pesos dos cordeiros do nascimento aos 210 dias foram positivas, variando de 0,19 a 0,64. Correlações entre pesos mais próximos foram mais altas. Martin et al. (1980), em uma revisão de estimativas para parâmetros de pesos de ovinos do nascimento aos 4 meses de idade, obtiveram a faixa de 0,5 a 1,0. Estas correlações sugerem que a seleção para um peso deve resultar em mudanças de pesos positivas para os outros pesos, mas pode causar aumento nos casos de distocia no parto. Este é da maior importância quando pouca seleção é colocada nas fêmeas e reprodutores de raças com altos pesos a maturidade são usados. Seleção para peso a desmama recebe mais ênfase quando os animais crescem e são terminados ao pasto (Rendel et al., 1969) e quando uma alta proporção de custos ocorre por causa da alimentação de animais em crescimento a ênfase é colocada em crescimento após desmama. As correlações fenotípicas entre pesos ao nascer até aos 210 dias foram, em geral, intermediárias e positivas $(0,21-0,39)$.

Verificou-se que a herdabilidade foi de 0,17 para o número de crias (repetibilidade 0,23). Land et al. (1983) relataram herdabilidade e repetibilidade desta característica de 0,11 e 0,15, enquanto Costa et al. (1990) acharam uma repetibilidade de 0,23 e Carillo \& Mocochá (1990), herdabilidade de 0,50.

\section{Conclusões}

A seleção de ovinos para peso à desmama deve resultar em aumento de peso em todas as idades. Cuidados devem ser tomados com o aumento de peso ao nascer devido a possível aumento em distocia. A herdabilidade materna na fase pré-desmama mostrou a importância da mãe no desenvolvimento do cordeiro nesta fase, bem como a importância da inclusão deste fator no modelo de análise.

A raça SI e os mestiços apresentaram pesos maiores que a raça MN. Quando os cordeiros começam a pastar, verifica-se que os mestiços passam a ter vantagem, mas os mestiços apresentaram maior intervalo de partos. Existe a necessidade de novos estudos de caráter econômico para a raça Morada Nova, principalmente sobre o seu uso em sistemas de cruzamento para a região.

\section{Literatura Citada}

BARBOSA, O.R.; SILVA, R.G.; SCOLAR, J. et al. Utilização de um índice de conforto térmico em zoneamento bioclimático da ovinocultura. In: REUNIÃO ANUAL DA SOCIEDADE BRASILEIRA DE ZOOTECNIA, 32., 1995, Brasília. Anais... Brasília: Sociedade Brasileira de Zootecnia, 1995. p.131-141.

BOLDMAN, K.G.; KRIESE, L.A.; VAN VLECK, L.D. et al. A manual for use of MTDFREML. A set of programs to obtain estimates of variances and covariances [DRAFT]. Washington: USDA, ARS, 1995. 120p. 
CARILLO, A.M.; MOCOCHÁ, C.E. Índice de herencia para número de corderos nacidos. In: CONGRESSO NACIONAL DE PRODUCCIÓN OVINO, 3., 1990, Tlaxcala. Memória... Tlaxcala: 1990. p.60-61.

CONSELHO NACIONAL DE DESENVOLVIMENTO CIENTÍFICO E TECNOLÓGICO - CNPq. Ovinocultura no Distrito Federal In: WORKSHOP DE OVINOCAPRINOCULTURA DO DISTRITO FEDERAL, 1., 1998. Planaltina. Workshop... Planaltina: 1998. 3p.

CONSELHO NACIONAL DE DESENVOLVIMENTO CIENTÍFCO E TECNOLÓGICO - CNPq. Ovinocultura no Ceará. In: WORKSHOP SOBRE CAPRINOS E OVINOS TROPICAIS, 1., 1999, Fortaleza. Workshop... Fortaleza: 1999. 83p.

COMBELLAS, J. Production and reproduction parameters of tropical sheep breeds in improved production systems. Tropical Animal Production, v.5, p.266-272, 1980.

COSTA, M.J.R.; QUEIROZ, S.A.; RIBEIRO, J.L.C. Avaliação de alguns aspectos do desempenho de ovinos da raça Morada Nova na região de Franca, SP. Revista da Sociedade Brasileira de Zootecnia, v.19, n.4, p.340-346, 1990.

FERNANDES, A.A.O.; BUCHANAN, D.S.; CASANOVA, H.S. Efeitos genéticos e de ambiente influenciando crescimento e sobrevivência de cordeiros Morada Nova. In: REUNIÃO DA SOCIEDADE BRASILEIRA DE ZOOTECNIA, 30., 1993, Rio de Janeiro. Anais... Rio de Janeiro: Sociedade Brasileira de Zootecnia, 1993. p.279.

FERNANDES, A.A.O.; SANDERS, J.O.; SHELTON, M. et al. 1985 Desempenho produtivo de um rebanho de ovinos Morada Nova no sertão do Ceará. In: REUNIÃO ANUAL DA SOCIEDADE BRASILEIRA DE ZOOTECNIA, 22., 1985, Camboriú. Anais... Camboriu: Sociedade Brasileira de Zootecnia, 1985. p.252.

FIGUEIREDO, E.A.P.; SIMPLÍCIO, A.A.; LIMA, F.A.M. et al. Estudo comparativo do crescimento de ovinos de diferentes raças no período de aleitamento. In: REUNIÃO DA SOCIEDADE BRASILEIRA DE ZOOTECNIA, 22., 1985, Camboriú. Anais... Camboriú: Sociedade Brasileira de Zootecnia, 1985. p.247.

FURUSHO, I.F.; PEREZ, J.R.O.; OLIVEIRA, M.V.M. Avaliação do desenvolvimento de cordeiros Santa Inês e cruzas Texel x Santa Inês e Texel $x$ Bergamácia até o desmame. In: REUNIÃO ANUAL DA SOCIEDADE BRASILEIRA DE ZOOTECNIA, 32., 1995. Brasília. Anais... Brasília: Sociedade Brasileira de Zootecnia, 1995. p.441-443.

INSTITUTO BRASILEIRO DE GEOGRAFIA E ESTATÍSTICA-IBGE. Anuário Estatístico do Brasil. Rio de Janeiro: 1995. v.57.

LAND, R.B.; ATKINS, K.D.; ROBERTS, R.C. Genetic improvement of reproductive performance. In: HARESIGN, W. (Ed.) Sheep production. London: Butterworths, 1983. p.515-525.

MARTIN, T.G.; SALES, D.I.; SMITH, C. et al. Phenotypic and genetic parameters for lamb weights in a synthetic line of sheep. Animal Production, v.18, p.237-247, 1980.

McMANUS, C.; MIRANDA, R.M. Crescimento de ovinos Bergamácia em Brasília. Parâmetros genéticos e fenotípicos e influências ambientais. Revista da Sociedade Brasileira de Zootecnia, v.25, n.4, p.627-635, 1996.
McMANUS, C.; MIRANDA, R.M. Comparação das raças de ovinos Santa Inês e Bergamácia no Distrito Federal. Revista da Sociedade Brasileira de Zootecnia, v.26, n.5, p.10551059, 1997.

McMANUS, C.; MIRANDA, R.M. Estimativas de parâmetros genéticos em ovinos Bergamácia, Revista da Sociedade Brasileira de Zootecnia, v.27, n.5, p.216-221, 1998.

RENDEL, J.M.; ALEXANDER, G.I.; WILLIAMS, L.G. et al. Selection of beef cattle breeding stock: Report of expert panel. Journal Australian Institute of Agricultural Science, v.34, p.28-31, 1968.

RODA, D.S.; SANTOS, L.E.; CUNHA, E.A. Peso ao nascer e mortalidade pré -desmame em cordeiros das raças Ideal e Corriedale. Boletim Industria Animal, v.52, p.67-70, 1995.

SAS INSTITUTE. User's guide: Statistics. Version 6,10 edition.Cary: 1995. 295p.

SILVA, F.L.R.; ARAUJO, A.M.; FIGUEIREDO, E.A.P. Características de crescimento e reprodução em ovinos Somalis no Nordeste Brasileiro. Revista Brasileira de Zootecnia, v.27, n.4, p.1107-1114, 1998.

SILVA, F.L.R.; FIQUEIREDO, E.A.P.; BARBIERI, M.E. et al. Efeito de ambiente e de reprodutor sobre as características de crescimento e reprodução em ovinos Santa Inês no Estado do Ceará. Revista da Sociedade Brasileira de Zootecnia, v.24, n.4, p.559-569, 1995.

SILVA, F.L.R.; FIQUEIREDO, E.A.P.; SIMPLICIO, A.A. Desempenho e parâmetros genéticos e fenotípicos para pesos e ganhos de peso pré-desmama, em cordeiros Santa Inês, no Ceará. In: REUNIÃO ANUAL DA SOCIEDADE BRASILEIRA DE ZOOTECNIA, 30., 1993, Rio de Janeiro. Anais... Rio de Janeiro: Sociedade Brasileira de Zootecnia, 1993a. p.276.

SILVA, F.L.R.; MILAGRES, J.C.; LIMA, E.A.M. Efeito de fatores genéticos sobre o crescimento pré- desmama em mestiços Santa Inês no Estado do Ceará. Pesquisa Agropecuária Brasileira, v.28, n.5, p.627-633, 1993b.

SOUSA, W.H.; PEREIRA, C.S.; BERGMANN, J.A.G. et al. Estimativas de componentes de (co)variância e herdabilidade direta e materna de pesos corporais em ovinos da raça Santa Inês. Revista Brasileira de Zootecnia, v.28, n.6, p.1252$1262,1999$.

TORRES, A.; MURAYI, P. Production characteristics of African long-fat-tailed sheep in Rwanda. Small Ruminant Research, v.1, p.108-115, 1987.

TOSH, J.J.; KEMP, R.A. Estimations of variance components for lamb weights in three sheep populations. Journal of Animal Science, v.72, p.1184-1191, 1994. 